\title{
HEPARIN ANTIBODIES AND THROMBOEMBOLISM IN HEPARIN-COATED AND NONCOATED VENTRICULAR ASSIST DEVICES
}

Andreas Koster, $\mathrm{MD}^{\mathrm{a}}$

Matthias Loebe, $\mathrm{MD}, \mathrm{PhD}^{\mathrm{b}}$

Ralf Sodian, $\mathrm{MD}^{\mathrm{b}}$

Evgenij V. Potapov, MD

Roland Hansen, $\mathrm{MD}^{\mathrm{c}}$

Johannes Müller, MD

Fritz Mertzlufft, $\mathrm{MD}, \mathrm{PhD}^{\mathrm{d}}$

George J. Crystal, $\mathrm{PhD}^{\mathrm{e}}$

Herrmann Kuppe, $\mathrm{MD}, \mathrm{PhD}^{\mathrm{a}}$

Roland Hetzer, $\mathrm{MD}, \mathrm{PhD}^{\mathrm{b}}$
Objective: Coating of ventricular assist devices (VADs) with heparin improves the biocompatibility and may reduce the need for systemic anticoagulation. However, heparins are associated with the risk of formation of heparin/platelet factor 4 antibodies (HPF4/A) and the development of heparin-associated thromboemboli. We analyzed the occurrence of HPF4/A and thromboembolism in patients with heparin-coated and noncoated VADs.

Methods: One hundred patients were enrolled in the investigation. Fiftyseven received a heparin-coated (group 1) and 43 a noncoated (group 2) VAD. HPF4/A testing was performed before and 2 and 12 weeks after implantation by the heparin platelet factor 4 enzyme-linked immunosorbent assay.

Results: There was no significant difference in the occurrence of HPF4/A in the 2 groups $(P=.102)$. Before the operation, 21 of the patients in group 1 had positive test responses and 25 in group 2. No patient had HPF4/A after termination of systemic heparinization. In group 1 there was no significant difference in the incidence of recurrent pump thromboses in patients who had positive test responses for HPF4/A $(n=11)$ when compared with those who had negative test responses $(\mathrm{n}=9, P=.89)$. Twenty-one patients had HPF/A but no thromboembolism. However, all 22 patients who had thromboembolism had HPF4/A.

Conclusions: Heparin coating of the VAD surface does not enhance the occurrence of HPF4/A-associated immunologic or thrombogenic reactions. However, the presence of these antibodies is strongly associated with an increased risk of thromboembolism in patients with a VAD. ( $\mathrm{J}$ Thorac Cardiovasc Surg 2001;121:331-5)
From the Departments of Anesthesiology $y^{\mathrm{a}}$ and Cardiothoracic and Vascular Surgery, ${ }^{\mathrm{b}}$ Deutsches Herzzentrum Berlin; Institute of Laboratory Medicine and Pathobiochemistry, ${ }^{\mathrm{c}}$ Campus Virchow Klinikum, Charité, Berlin; Department of Anesthesiology and Intensive Care Medicine, ${ }^{\mathrm{d}}$ University of Homburg, Saar, Germany; Department of Anesthesiology, e Illinois Masonic Medical Center; and the Departments of Anesthesiology and Physiology and Biophysics, University of Illinois College of Medicine, Chicago, Ill.

Received for publication Feb 1, 2000; revisions requested April 11, 2000; revisions received May 8, 2000; accepted for publication Sept 5, 2000.

Address for reprints: Andreas Koster, MD, Deutsches Herzzentrum Berlin, Augustenburger Platz 1, D-13353 Berlin, Germany (Email: koster@dhzb.de).

Copyright (c) 2001 by The American Association for Thoracic Surgery

0022-5223/2001 $\$ 35.00+0 \quad \mathbf{1 2 / 1 / 1 1 1 6 5 5}$

doi:10.1067/mtc.2001.111655
/ entricular assist devices (VADs), because of their large foreign inner surface and heart valves, encourage the formation of thrombi, which could become dislodged, enter the circulation, and travel to the various body tissues with potentially devastating consequences. ${ }^{1}$ One approach to prevent this problem is the use of high doses of anticoagulants. However, this technique has the risk of bleeding complications. Therefore, biocompatible inner surfaces have been developed for use in VADs to reduce the tendency for thrombus formation and consequently to enable a reduction of systemic anticoagulation. In the HeartMate left ventricular assist device (LVAD; Thermo Cardiosystems Inc, Woburn, Mass), this has been achieved by the creation of a surface on which a pseudoendothelium layer is 
generated. In the Berlin Heart VAD (Mediport, Berlin, Germany), the inner surface of the pumps and cannulas are coated with unfractionated heparins. The coating is done by the CARMEDA process (Carmeda, Sa, Lund, Sweden), which has been successfully used for coating cardiopulmonary bypass (CPB) circuits to reduce contact activation of the coagulation system, the need for systemic anticoagulation, and the inflammatory response to the perfusion. ${ }^{2-4}$ A possible disadvantage of coating VADs with unfractionated heparins is an enhanced tendency for the formation of heparin/platelet factor 4 antibodies (HPF4/A), which have been demonstrated to play a pivotal role in the pathogenesis of heparin-induced thrombocytopenia type II (HIT II). ${ }^{5}$ HIT II is characterized by the induction of antibodies against complexes of heparin and platelet factor 4 . The newly developed antigen-antibody complex binds to platelets and initiates a cascade of events, which result in excessive platelet aggregation that finally leads to severe thromboembolic complications. ${ }^{6,7}$

The goal of the present clinical investigation was as follows:

1. To evaluate whether coating VADs with unfractionated heparin affects the occurrence of HPF4/A

2. To determine whether this is associated with an increased risk for the development of thromboembolic complications, in particular on coated surfaces, in these patients.

\section{Methods}

Devices. The Berlin Heart VAD consists of a polyurethane blood pump that is coated with unfractionated heparins (Carmeda). Each pump carries 2 mechanical heart valves and is connected by 2 silicone cannulas. The Berlin Heart system can be used for both single ventricular and biventricular assistance. The LVAD (Baxter Healthcare Corp, Oakland, Calif) is made of polyurethane and connected by Dacron inflow and outflow tracts. It contains biologic heart valves. The TCI HeartMate LVAD chamber (Thermo Cardiosystems) is made of titanium with a rough inner layer, which permits the generation of a natural pseudoendothelium. The DeBakey LVAD (Micromed Technology, the Woodlands, Tex) generates a laminar flow by a rotary blood pump and uses a valveless Dacron outflow graft.

Groups of patients. After approval by the local ethics committee and informed consent, 100 patients who received a VAD in our institution from 1995 to 1999 were enrolled in the investigation. The investigation was performed by using deep-frozen samples, which were collected during hospitalization of the patients. The decision to implant an LVAD or a biventricular assist device system was based on clinical necessity (cardiogenic shock and severe right heart failure). A Berlin Heart system was implanted if the patient needed biventricular assistance. A solely left ventricular Berlin Heart system was planted via a left thoracotomy in patients who had undergone median sternotomy previously. Patients were divided into 2 groups. Group 1 consisted of patients with a heparin-coated VAD (Berlin Heart system), and group 2 consisted of patients with a noncoated VAD (other systems).

Anticoagulation protocol. Before the operation, anticoagulation was switched to unfractionated heparin (UFH; Liquemin) to achieve a target activated partial thromboplastin time value between 40 and 60 seconds. Anticoagulation during implantation with the use of CPB was accomplished with UFH according to the Hepcon HMS (Medtronic HemoTec, Inc, Englewood, Colo; E. M. Parker Company, Inc, Wilmington, Mass). After termination of $\mathrm{CPB}$, the anticoagulant effect of heparin was reversed with protamine sulfate (Hoffmann-La Roche Ltd, Basel, Switzerland). Intravenous heparinization was restarted 6 to 12 hours after the operation to achieve a target activated clotting time of 160 to 180 seconds. Antiplatelet agents were simultaneously administered on the basis of the results of the in vitro platelet aggregation tests. The tests were performed with $20 \mu \mathrm{mol} / \mathrm{L}$ adenosine diphosphate, $100 \mu \mathrm{L} / \mathrm{L}$ epinephrine, $190 \mu \mathrm{g} / \mathrm{mL}$ collagen, and $500 \mu \mathrm{g} / \mathrm{mL}$ arachidonic acid. The target value for adenosine diphosphate- and epinephrine-induced platelet aggregation was $30 \%$ to $50 \%$ and less than $40 \%$ for arachidonic acid-induced aggregation. A decrease of the aggregation to a value of $70 \%$ to $90 \%$ was regarded as sufficient for collagen-induced platelet aggregation. Antiplatelet therapy was initiated with aspirin, beginning with $50 \mathrm{mg} / \mathrm{d}$ up to 200 $\mathrm{mg} / \mathrm{d}$ as a maximum dose. If patients did not respond to this therapy, dipyridamole (400-1000 mg/d) was given.

After removal of all drainage tubes, patients with the Berlin Heart, Novacor, and DeBakey LVADs were switched to warfarin sodium and aspirin, depending on the results of the platelet function test. Patients on the TCI system were treated with aspirin only. Heparinization was only re-established in cases of an increased risk for thrombus formation (immobilization, parameters of infection as demonstrated by white blood cell count, and measurement of C-reactive protein) and a tendency for hyperaggregation in the platelet aggregation assay, which did not respond to antiplatelet therapy.

Detection of thromboembolism. Thromboembolism was confirmed or excluded by computed tomography, angiography, and Doppler ultrasonography after clinical suspicion. In the Berlin Heart VAD the pumps are placed outside the patient, and because they are transparent, visual examination of the pumps for thrombus formation was performed daily. Thrombus formation was regarded as recurrent if they were found in the pumps on more than 3 occasions during the 3month period of the investigation.

Detection of HPF 4/A. Blood samples were collected before implantation, on the seventh and fourteenth postoperative days, and 3 months later. The samples were tested by the use of the ASSERCHROM HPIA enzyme immunoassay (Diagnostica Stago, Asnierssur-Seine, France). The results were considered positive if an optical density of greater than 0.480 was found at an absorbency level of $492 \mathrm{~nm}$.

Platelet count and heparin-induced platelet aggregation assay. The platelet count was performed daily. The heparin 
platelet aggregation assay (HIPAA) ${ }^{8}$ was performed if the patient had a postoperative platelet count of less than $100,000 / \mathrm{mL}$ that failed to recover within 2 days or if a patient had a decrease in the platelet count of greater than $30,000 / \mathrm{mL}$. The HIPAA result was deemed positive when agglutination was observed in 2 of 4 chambers with 0.2 $\mathrm{IU} / \mathrm{mL}$ heparin and a negative result in the buffer and the 100 $\mathrm{IU} / \mathrm{mL}$ heparin chambers. In patients who had positive test results for HIT II according to the HIPAA, further anticoagulation was performed with recombinant hirudin (Refludan), with a target activated partial thromboplastin time value of 40 to 60 seconds. ${ }^{9}$

Statistical analysis. Statistical analyses of the data were performed with SPSS 7.5 for Windows (SPSS, Inc, Chicago, Ill). Significant differences were confirmed by the Student $t$ test (for continuous data). Qualitative variables were analyzed by the $\chi^{2}$ test.

\section{Results}

There were no significant differences in the biometric data, parameters of infection, and duration of heparinization for the 2 groups. Fifty-five patients belonged to group 1 and 45 to group 2 . There were 64 male and 36 female patients, with ages ranging from 18 to 64 years (mean $\pm \mathrm{SD}, 47 \pm 10$ years). In 63 patients the implantation of the device was necessary because of dilated cardiomyopathy, in 5 because of restrictive cardiomyopathy, in 7 because of acute myocarditis, and in 25 because of ischemic cardiomyopathy. In 51 patients the implantation of a biventricular assist device (Berlin Heart) was necessary. Forty-nine patients received an isolated LVAD (30 Novacor, 7 TCI, 6 Berlin Heart, and 6 DeBakey VAD).

Prevalence and persistence of HPF4/A. In group 1, 32 patients had positive test responses for HPF4/A (21 before the operation), whereas in group 2, 31 patients had positive test results for HPF4/A (25 before the operation, $P=.102$ ). No patient had positive results for HPF4/A after discontinuation of heparin treatment. Fourteen patients from group 1 and 18 patients from group 2 had negative results for HPF4/A after discontinuation of heparin. Thirteen patients from group 1 and 14 patients from group 2 continued to have positive results for HPF4/A after discontinuation of heparin. There were no significant differences in the prevalence and persistence of HPF4/A between the 2 groups.

HPF4/A and embolism. Embolic events (cerebral infarction, mesenteric infarction, pulmonary embolism, and peripheral embolization of the extremities) were identified in 10 patients with positive results for HPF4/A in group 1 and 12 with positive results in group 2. There were no thromboembolic events in patients with negative results for HPF4/A. Twenty-two patients in group 1 and 19 in group 2
Table I. HPF4/A in heparin-coated and noncoated VADs

\begin{tabular}{lcc}
\hline & Heparin-coated & Noncoated \\
\hline Patient total & 57 & 43 \\
Positive results & 32 & 31 \\
$\begin{array}{l}\text { First day positive } \\
\begin{array}{l}\text { Became positive after intravenous } \\
\quad \text { heparin stopped }\end{array}\end{array}$ & 21 & 25 \\
$\begin{array}{l}\text { Became negative after intravenous } \\
\quad \text { heparin stopped }\end{array}$ & 14 & 0 \\
\hline
\end{tabular}

Table II. HPF4/A and thromboembolism

\begin{tabular}{lcc}
\hline & Heparin-coated & Noncoated \\
\hline Positive with TE & 10 & 12 \\
Positive without TE & 22 & 19 \\
Negative without TE & 0 & 0 \\
\hline
\end{tabular}

$T E$, Thromboembolism.

with negative results for HPF4/A had no occurrence of emboli. There were no significant differences between the 2 groups.

Recurrent pump thromboses and HPF4/A in heparin-coated VADs. In group 1, 11 patients with positive results for HPF4/A and 9 patients with negative results for the antibody exhibited at least 3 repetitive thrombi in the pump. There was no significant difference between the occurrence of repetitive pump thrombus formation for patients with positive and negative HPF4/A results. Twenty-one patients with positive results for HPF4/A had no repetitive thrombus formation in the pump.

Platelet count and HIPAA. The HIPAA was performed in 13 patients: 7 from the coated group and 6 from the noncoated group. Three of these patients had a positive reaction, and in all of these, HPF4/A was detectable in the enzyme-linked immunosorbent assay. One of these 3 patients demonstrated no clinical symptoms of thromboembolism, 1 had a stroke caused by embolization, and the third had acute Leriche syndrome. Three of the 10 patients with a negative HIPAA result had positive results for HPF/4A. There were no significant differences in platelet count and HIPAA for groups 1 and 2.

\section{Discussion}

The main findings from our study were that heparin coating of the VAD surface does not enhance HPF4/Aassociated immunologic or thrombogenic reactions. However, the presence of these antibodies is strongly associated with an increased risk of thromboembolism in patients with a VAD. This is of great importance in 
Table III. Recurrent pump thromboses and HPF4/A in coated VADs

\begin{tabular}{lc}
\hline & Patients \\
\hline Total & 57 \\
HPF $4 / A^{+}$ & 32 \\
rPT/HPF $4 / A^{+}$ & 11 \\
rPT/HPF4/A & 9 \\
HPF $4 / A^{+}$, no rPT & 21 \\
\hline
\end{tabular}

$r P T$, Recurrent pump thrombosis.

view of the comparably high prevalence $(63 \%)$ of antibodies in these patients.

HPF4/A plays a pivotal role in HIT II and in the formation of heparin-associated thromboemboli. Large differences have been described in the incidence of these antibodies in patients who undergo different types of operations. Bauer and associates ${ }^{10}$ found HPF4/A in $19 \%$ of patients before and $51 \%$ after cardiac operations, whereas Trossaért, ${ }^{11}$ Pouplard, ${ }^{12}$ and their colleagues found it in $27 \%$ and $25.3 \%$ of patients, respectively, after cardiac operations. Visentin and coworkers ${ }^{13}$ described an incidence of HPF4/A of $61 \%$ after cardiac operations and $4.5 \%$ after noncardiac operations; they reported no HPF4/A in patients who did not have operations. Jackson and associates ${ }^{14}$ reported an incidence of HPF4/A in $2 \%$ of patients who underwent vascular operations. The large fraction of patients who revealed HPF4/A before operations in the present study may be explained by multiple hospitalizations and exposures to heparin before the need for implantation of a VAD.

In our study only patients with positive results for HPF4/A had thromboemboli and clinical symptoms of HIT II. Thus the presence of such antibodies may be regarded as a predictor of an increased risk for the development of thromboembolic complications.

Several findings from this study suggest that coating of the inner surface of VADs with unfractionated heparins does not lead to an immunization against heparins. First, no patient from group 1 (the coated group) had antibodies after reversal of systemic heparinization. Second, there was no difference in the 2 groups in the number of patients whose results became antibody negative after the termination of systemic heparinization or in the incidence of thromboembolic complications. Furthermore, circulating antibodies seemed not to induce thrombus formation on the heparinized surface because there was no difference in the incidence of repetitive pump thrombi between patients with a positive antibody status when compared with patients with a negative status in the heparin-coat- ed group. These clinical data are consistent with the results of recent laboratory investigations, which revealed that HPF4/A does not bind to platelet factor 4 complexed with heparin immobilized by multiple chemical cross linkages. ${ }^{15}$ However, further investigations to prove whether this also applies for other Carmeda-coated materials, such as CPB systems or catheters, are required.

In conclusion, we found that patients with a VAD have a high incidence of HPF4/A. These antibodies were strongly associated with an increased risk of development of thromboembolic complications, and the frequency of these complications was independent of whether the inner surface of VAD was coated with heparin. Further investigations are necessary to clarify the precise role played by the HPF4/A in thrombus and embolus formation.

We thank Tonie Derwent for professional assistance in the editing of the manuscript and Julia Stein for her assistance with the statistics.

\section{REFERENCES}

1. Himmelreich G, Ullmann H, Riess H, Rosch R, Leobe M, Schiessler U, et al. Pathophysiologic role of contact activation in bleeding followed by thromboembolic complications after implantation of a ventricular assist device. ASAIO J 1995;41:M790-4.

2. Jansen PG, te Velthuis H, Huybregts RA, Paulus R, Bulder ER, van der Spoel HI, et al. Reduced complement activation and improved postoperative performance after cardiopulmonary bypass with heparin-coated circuits. J Thorac Cardiovasc Surg 1995; 110:829-34.

3. te Velthuis H, Baufreton C, Jansen PG, Thijs CM, Hack CE, Sturk A, et al. Heparin coating of extracorporeal circuits inhibits contact activation during cardiac operations. J Thorac Cardiovasc Surg 1997;114:117-22.

4. Fukotomi M, Kobayashi S, Niwaya K, Hamada Y, Kitamura S. Changes in platelet, granulocyte, and complement activation during cardiopulmonary bypass using heparin coated equipment. Artif Organs 1996;20:767-76.

5. Amiral J, Bridey F, Dreyfuss M, Vissac AM, Fressinaud E, Wolf $\mathrm{M}$, et al. Platelet factor 4 complexed to heparin is the target for antibodies generated in heparin-induced thrombocytopenia. Thromb Haemost 1992;68:95-6.

6. Slaughter TF, Greenberg CS. Heparin-induced thrombocytopenia and thrombosis implications for perioperative management. Anesthesiology 1997;87:667-75.

7. Shorten GD, Communale ME. Heparin-induced thrombocytopenia. J Cardiothorac Vasc Anesth 1996;10:521-30.

8. Greinacher A, Michels I, Kiefel V, Mueller Eckard C. A rapid and sensitive test for diagnosing heparin-induced thrombocytopenia. Thromb Haemost 1991;66:734-6.

9. Greinacher A, Volpel H, Janssens U, Hach-Wunderle V, KemkesMattes B, Eichler P, et al. Recombinant hirudin (lepirudin) provides safe and effective anticoagulation in patients with heparin- 
induced thrombocytopenia: a prospective study. Circualtion 1999;99:73-80.

10. Bauer TL, Arepally G, Konkle BA, Mestichelli B, Shapiro SS, Cines DB, et al. Prevalence of heparin-associated antibodies without thrombosis in patients undergoing cardiopulmonary bypass surgery. Circulation 1997;95:1242-6.

11. Trossaért M, Gaillard A, Commin PL, Amiral J, Vissac AM, Fressinaud E. High incidence of antiheparin/platelet factor 4 antibodies after cardiopulmonary bypass surgery. $\mathrm{Br} \mathrm{J}$ Haematol 1998;101:653-5.

12. Pouplard C, May MA, Iochmann S, Amiral J, Vissac AM, Marchand M, et al. Antibodies to platelet factor 4-heparin after cardiopulmonary bypass in patients anticoagulated with unfractionated heparin or a low-molecular-weight heparin: clinical implications for heparin-induced thrombocytopenia. Circulation 1999;99:2530-6.

13. Visentin GP, Malik M, Cyganiak KA, Aster RH. Patients treated with unfractionated heparin during open heart surgery are at high risk to form antibodies with heparin: platelet factor 4 complexes. J Lab Clin Med 1996;128:376-83.

14. Jackson MR, Gillespie DL, Chang AS, Longenecker EG, Peat RA, Alving BM. The incidence of heparin-induced antibodies in patients undergoing vascular surgery: a prospective study. J Vasc Surg 1998;28:439-44.

15. Suh JS, Aster RH, Visentin GP. Antibodies from patients with heparin-induced thrombocytopenia/thrombosis recognize different epitopes on heparin: platelet factor 4. Blood 1998;91:916-22.

\section{Authoritative}

The Journal of Thoracic and Cardiovascular Surgery is the most frequently cited thoracic/cardiovascular surgery journal in the Science Citation Index. An article in JTCVS is sited on average almost twice as often as those in the closest cardiothoracic journal. 